\title{
Quantum Critical Behavior in Gauged Yukawa Matrix Field Theories with Quenched Disorder
}

\author{
H. Hamidian \\ May 12, 2018 \\ Department of Physics, Stockholm University, Box 6730, S-113 85 Stockholm, Sweden

\begin{abstract}
We use the Wilson-Fisher $\epsilon$ expansion to study quantum critical behavior in gauged Yukawa matrix field theories with weak quenched disorder. We find that the resulting quantum critical behavior is in the universality class of the pure system. As in the pure system, the phase transition is typically first order, except for a limited range of parameters where it can be second order with computable critical exponents. Our results apply to the study of two-dimensional quantum antiferromagnets with weak quenched disorder and provide an example for fluctuation-induced first order phase transitions in circumstances where naively none is expected.
\end{abstract}


One of the most remarkable features of quantum spin systems is their relationship to gauge theories. This connection, which was originally used to study chiral symmetry breaking in QCD in the strong coupling limit [四], remains a powerful tool. Recently this analogy has been used to prove that certain gauge theories break chiral symmetry in the strong coupling limit [2-4]. Also, it has been further suggested [5] that the underlying analogy can be used in a much broader sense to account for the quasi-particle spectrum and other infrared features of two-dimensional (2D) quantum antiferromagnets and three-dimensional non-Abelian gauge theories. Using the quantum chromodynamics (QCD) terminology, the quantum phase transition with a chiral symmetry breaking pattern is then characterized by the number of flavors $\left(N_{F}\right)$ and colors $\left(N_{C}\right)$ of quarks in the gauge theory. In the antiferromagnet the rank of the spin algebra and the size of its representation play the same role as the number of flavors and colors respectively.

The study of $2 \mathrm{D}$ quantum antiferromagnets, particulary in connection with high- $T_{C}$ superconductivity, is a rapidly developing subject of great current interest [6]. An important question that naturally arises is the stability of the antiferromagnetic long-range order (LRO) in the presence of quenched disorder (QD). In fact, since the Néel temperature of planar high$T_{C}$ superconductors, such as $\mathrm{La}_{2} \mathrm{CuO}_{4}$, is extremely sensitive to impurities and defects, it is probably not correct to ignore the impurity effects even in high-quality samples used in laboratories. The critical behavior of low-dimensional systems with quenched disorder and an $O(N)$-vector magnetic order parameter has been addressed by a number of authors [7, 8] by using a Landau-Ginzburg-Wilson (LGW) Hamiltonian and applying the Wilson-Fisher (WF) $\epsilon$ expansion [9]. In particular, Boyanovsky and Cardy [8] have carried out a two-loop (double) $\epsilon$ expansion in systems in which the impurities are correlated in $\epsilon_{d}$ dimensions and randomly distributed in $d-\epsilon_{d}$ dimensions. They take the full structure of the theory into account which, as a result of anisotropies, leads to highly nonlocal interactions.

In this paper we shall study the stability of LRO in 2D quantum antiferromagnets with QD by using the WF $\epsilon$ expansion and renormalization group (RG) techniques. We shall approach this problem by examining the critical behavior of the related zero temperature gauged Yukawa matrix field theories in three (Euclidean) dimensions in the presence of quenched disorder. This requires that the anisotropies be properly taken into account, as is done (see Ref. [8], e.g.) for the $O(N)$-vector model in the presence of QD. However we shall go one step further and also examine the stability of the theory against quantum fluctuations which can arise through the Coleman-Weinberg mechanism [10]. Our results enable us to argue that below a certain critical doping (impurity concentration) $\Delta_{\text {crit. }}$, and in a narrow region in parameter space, $2 \mathrm{D}$ doped quantum antiferromagnets belong to the universality class of gauged Yukawa matrix field theories which describe the pure system. We check this result through an explicit calculation of the critical exponent $\nu$ and find that the Harris criterion [11] is indeed satisfied. However, we find that a careful examination of the RG flows in the space of couplings indicates that LRO can be destroyed due to quantum fluctuations. This means that there are cases where there is an infrared (IR) stable fixed point and the Harris criterion is satisfied, but the phase transition is fluctuation-induced first order rather than second order, contrary to what is naively expected. We shall begin with a brief review of some recent work on pure quantum antiferromagnets and gauged Yukawa matrix theories. 
The pure 2D (generalized) quantum antiferromagnet is defined by the Hamiltonian

$$
H_{\text {spin }}=\kappa \sum_{<x, y>} \sum_{A=1}^{N_{F}^{2}-1} J^{A}(x) J^{A}(y)
$$

with $\langle x, y\rangle$ nearest neighbor sites $x$ and $y$ on a square lattice and the "spin" operators $J^{A}(x)$ in an irreducible representation of the $S U\left(N_{F}\right)$ Lie algebra

$$
\left[J^{A}(x), J^{B}(y)\right]=i f^{A B C} \delta(x, y) J^{C}(x)
$$

When the representation at each site is a rectangular Young Tableau with $m$ rows and $N_{C}$ columns, it is convenient to represent the spin operators by the fermion bilinears

$$
J^{A}(x)=\sum_{\alpha=1}^{N_{C}} \sum_{a, b=1}^{N_{F}} \psi_{a \alpha}^{\dagger}(x) T_{a b}^{A} \psi_{b \alpha}(x)
$$

The fermions have the anticommutator,

$$
\left\{\psi_{a \alpha}(x), \psi_{b \beta}^{\dagger}(y)\right\}=\delta_{a b} \delta_{\alpha \beta} \delta(x, y)
$$

Constraints which project out the irreducible representation of the spin algebra are

$$
\mathcal{G}_{\alpha \beta}(x) \equiv \sum_{a=1}^{N_{F}} \psi_{a \alpha}^{\dagger}(x) \psi_{a \beta}(x)-\delta_{\alpha \beta} N_{F} / 2 \sim 0 \quad \forall x
$$

$\mathcal{G}_{\alpha \beta}(x)$ obeys the $U\left(N_{C}\right)$ Lie algebra, commutes with the Hamiltonian and acts as the generator of gauge transformations with gauge group $U\left(N_{C}\right)$.

The critical behavior of the antiferromagnet was examined by Read and Sachdev [12 using semiclassical methods. The only free parameters are the integers $N_{C}$ and $N_{F}$. $N_{C} \ll$ $N_{F}$ is the classical limit of large representations, where the classical Néel ground state is stable with the staggered spin order parameter

$$
\mu_{a b}=(-1)^{\sum_{i} x_{i}}<\sum_{\alpha=1}^{N_{C}} \psi_{a \alpha}(x) \psi_{b \alpha}(x)>
$$

On the other hand, the limit $N_{F} \ll N_{C}$ is the quantum limit where fluctuations are important and the system is in a spin disordered state. For both $N_{C}$ and $N_{F}$ large, they find a line of second order phase transitions in the $\left(N_{C}, N_{F}\right)$ plane at $N_{F}=$ const. $\cdot N_{C}$ where the constant is a number of order one.

The relationship between the antiferromagnet and QCD is a very close one. There is an argument in ref. [3] which maps the strong coupling limit of lattice QCD onto the quantum antiferromagnet. The lattice regularization of the QCD Hamiltonian uses staggered fermions [13]. Since the order parameters are identical, the Néel ordered states of the antiferromagnet correspond to chiral symmetry breaking states of QCD. Thus, the infinite coupling limit of QCD is identical to the quantum antiferromagnet. A main difference between QCD with finite coupling and the quantum antiferromagnet is that QCD allows a 
fermion kinetic energy term while retaining gauge invariance, whereas in the antiferromagnet, the fermions are not allowed to move. One could, however, regard the corrections to the strong coupling limit of QCD as the addition of degrees of freedom and gauge invariant perturbations in the quantum antiferromagnet which allow fermion propagation. In fact, it has even been suggested [5] that the additional degrees of freedom are generated dynamically.

A common feature of both theories is that, aside from $N_{F}$ and $N_{C}$ they have no free parameters. One could imagine adding operators of the sort that, if their coupling constants is varied, can induce the chiral phase transition. It is tempting to speculate that these transitions fall into a universality class which can take into account all such modifications, as long as they respect the symmetries of the theory. Restricting attention only to those operators which lead to a Lorentz invariant continuum limit, it was recently argued [14 that the universality class is described by the $4-\epsilon$ dimensional Euclidean field theory,

$$
\begin{aligned}
S= & \int d^{4-\epsilon} x\left[\frac{1}{2} \operatorname{tr}(\nabla \phi) \cdot(\nabla \phi)\right. \\
& +\frac{8 \pi^{2} \mu^{\epsilon}}{4 !}\left(\frac{g_{1}}{N_{F}^{2}}\left(\operatorname{tr} \phi^{2}\right)^{2}+\frac{g_{2}}{N_{F}} \operatorname{tr} \phi^{4}\right)+\frac{1}{4} \operatorname{tr} F_{\mu \nu}^{2} \\
& +\bar{\psi}\left(\gamma \cdot \nabla+i \mu^{\epsilon / 2} e_{1} \gamma \cdot A+i \mu^{\epsilon / 2} e_{2} \gamma \cdot \operatorname{tr} A\right. \\
& \left.\left.+\frac{\pi \mu^{\epsilon / 2} y}{\sqrt{N_{F} N_{C}}} \phi\right) \psi\right)
\end{aligned}
$$

Here the scalar field $\phi$ is an $N_{F} \times N_{F}$ traceless Hermitean matrix. The 4-component spinor $\psi$ is an $N_{F} \times N_{C}$ complex matrix and $A_{\mu}$ is a $U\left(N_{C}\right)$ gauge field. In four dimensions this model has Euclidean Lorentz invariance, C,P and T, discrete chiral symmetry, $\left(\psi \rightarrow \gamma^{5} \psi\right.$, $\phi \rightarrow-\phi)$ and global $S U\left(N_{F}\right)$ flavor. (7) includes all operators which are marginal when $D=4$.

The evidence that (7) describes the universality class comes from previous work [15] where a similar model where gauge couplings are absent was studied. In Ref. [14 it was shown that the anomalous dimensions of operators computed in the model (7) with $e_{i}=0$ were identical to leading order in $1 / N_{C}$ and $\epsilon$ to those of a $2<D<4$ dimensional fourfermi theory. That a $4-\epsilon$ dimensional Yukawa-Higgs theory has the same universal critical behavior as lower dimensional four-fermi theories with the same symmetries was originally suggested by Wilson [16]. For the case $N_{F}=1$, where the chiral symmetry is discrete, higher order computations have been carried out [17, 18]. These results, as well as those of lattice simulations, support the universality hypothesis [17]. It was argued in [14 that (7) represents the universality class of lower-dimensional four-fermi theories with $U\left(N_{C}\right)$ gauge invariance such that for a large range of values of $\left(N_{C}, N_{F}\right)$, the chiral phase transition is a fluctuation induced first order transition and when it is second order, critical exponents are in principle computable in the $\epsilon$ expansion.

To include the effects of QD amounts to adding new operators which can induce the chiral phase transition by varying the width, $\Delta$, of the impurity-probability distribution. This is achieved by adding

$$
\int d^{d} x(r+\delta r(x)) \operatorname{tr} \phi^{2}(x)
$$


to the aciton (7). Here $\delta r(x)$ is the impurity field and $r$ is the mass of the field $\phi(x)$ (renormalized to zero at the critical point). The quenched partition function can be written by using the replica trick [19]. Defining the impurity-probability distribution $P[\delta r]$ such that the replica average $\langle\langle\delta r(x)\rangle\rangle=0$ and

$$
\left\langle\left\langle\delta r(x) \delta r\left(x^{\prime}\right)\right\rangle\right\rangle=\Delta \delta^{d-\epsilon_{d}}\left(x-x^{\prime}\right),
$$

a cumulant expansion yields the (replica) action for the gauged Yukawa matrix field theory with QD

$$
\begin{aligned}
S_{\mathrm{QD}}= & \sum_{\alpha} S_{\alpha}-\frac{\Delta}{2} \int d^{d} x \int d^{d} x^{\prime} \delta^{d-\epsilon_{d}}\left(x-x^{\prime}\right) \\
& \sum_{\alpha} \operatorname{tr} \phi_{\alpha}^{2}(x) \sum_{\beta} \operatorname{tr} \phi_{\beta}^{2}\left(x^{\prime}\right) .
\end{aligned}
$$

Here $\alpha, \beta=1, \ldots, n$ are the replica indices and $S_{\alpha}$ has the same form as $S$ in (7) with a replica index $\alpha$ for each field operator and $d=4-\epsilon$. In (10) the impurities are correlated along $\epsilon_{d}$ dimensions, whereas in the remaining $d-\epsilon_{d}$ directions the impurity-correlation function (9) is zero for impurities at different points. It is important to note that the system is characterized by two dimensionalities, namely $\epsilon$ and $\epsilon_{d}$, which means that the perturbative expansion will, in general, involve a double $\epsilon$ expansion. Such a double $\epsilon$ expansion is necessary for a controlled perturbative description of the critical behavior (see Dorogovtsev in Refs. [7] and [8]).

To investigate the critical behavior we first compute the $\beta$ functions for the model defined by (10). To leading order, our calculations yield

$$
\begin{aligned}
& \beta_{1}=-\epsilon g_{1}+\frac{N_{F}^{2}+7}{6 N_{F}^{2}} g_{1}^{2}+\frac{2 N_{F}^{2}-3}{3 N_{F}^{2}} g_{1} g_{2}+\frac{N_{F}^{2}+3}{2 N_{F}^{2}} g_{2}^{2}+\frac{1}{2 N_{F}} y^{2} g_{1}-24 g_{1} \Delta, \\
& \beta_{2}=-\epsilon g_{2}+\frac{2}{N_{F}^{2}} g_{1} g_{2}+\frac{N_{F}^{2}-9}{3 N_{F}^{2}} g_{2}^{2}-\frac{3}{8 N_{C} N_{F}} y^{4}+\frac{1}{2 N_{F}} y^{2} g_{2}, \\
& \beta_{y}=-\frac{\epsilon}{2} y-\frac{3}{16 \pi^{2}} \frac{N_{C}^{2}-1}{N_{C}} e_{1}^{2} y-\frac{3}{8 \pi^{2}} e_{2}^{2} y+\frac{N_{F}^{2}+2 N_{C} N_{F}-3}{16 N_{C} N_{F}^{2}} y^{3}, \\
& \beta_{e_{1}}=-\frac{\epsilon}{2} e_{1}-\frac{11 N_{C}-2 N_{F}}{48 \pi^{2}} e_{1}^{3}, \\
& \beta_{e_{2}}=-\frac{\epsilon}{2} e_{2}+\frac{N_{C} N_{F}}{12 \pi^{2}} e_{2}^{3}, \\
& \beta_{\Delta}=-\tilde{\epsilon} \Delta-16 \Delta^{2}+\frac{N_{F}^{2}+1}{3 N_{F}^{2}} g_{1} \Delta+\frac{2 N_{F}^{2}-3}{3 N_{F}^{2}} g_{2} \Delta,
\end{aligned}
$$

where $\tilde{\epsilon}=\epsilon+\epsilon_{d}$ and a factor of $2 \pi^{\tilde{d}} \Gamma(\tilde{d} / 2)$, with $\tilde{d}=4-\tilde{\epsilon}$, has been absorbed into the definition of $\Delta$. Fixed points occur at the zeros of the $\beta$ functions, $\beta\left(g_{i}^{*}\right)=0$. The fixed points are IR stable if all the eigenvalues of the stability matrix, $\partial \beta_{i} /\left.\partial g_{j}\right|_{g=g^{*}}$, are positive. Second order phase transitions are possible when the RG trajectories flow to the IR stable fixed point in the region allowed by general stability conditions. Otherwise, the only allowed 
phase transition is a fluctuation induced first order one. Yamagishi [20] formulated a criterion for this behavior. He showed that this occurs when the RG trajectory crosses the surface in coupling-constant space given by

$$
\mathcal{P}_{i}(g, y, e)=0, \quad i=1,2,
$$

where $\mathcal{P}_{1}(g, y, e)=(4-\epsilon)\left(g_{1}+g_{2}\right)+\beta_{1}+\beta_{2}$ and $\mathcal{P}_{2}=(4-\epsilon)\left(g_{1}+\left(N_{F} / 2\right) g_{2}\right)+\beta_{1}+\left(N_{F} / 2\right) \beta_{2}$ depending on whether $g_{2}>0$ or $g_{2}<0$, respectively. When the RG trajectories cross these surfaces two further conditions must be met. To ensure that the extremum is a local minimum, rather than a maximum, it is necessary that $D_{i}>0$ for $i=1,2$ where $D_{1}=$ $(4-\epsilon)\left(\beta_{1}+\beta_{2}\right)+\Sigma_{i} \beta_{i} \partial / \partial g_{i}\left(\beta_{1}+\beta_{2}\right)$ or $D_{2}=(4-\epsilon)\left(\beta_{1}+\left(N_{F} / 2\right) \beta_{2}\right)+\Sigma_{i} \beta_{i} \partial / \partial g_{i}\left(\beta_{1}+\left(N_{F} / 2\right) \beta_{2}\right)$ depending on whether $g_{2}>0$ or $g_{2}<0$.

For this minimum to have lower free energy than the one at the trivial $\phi=0$, it is necessary that the couplings at that scale obey

$$
g_{1}+g_{2}<0 \text { or } g_{1}+N_{F} g_{2} / 2<0 .
$$

By using Eqns. (11)-(16) we have numerically solved for the RG trajectories in the sixdimensional space of couplings. In Fig. 1 we have plotted the projection of some of the RG trajectories onto the $g_{1}-g_{2}$ plane for the case $N_{C}=2, N_{F}=13$ and $\epsilon=\epsilon_{d}=0.1$. As in the pure case, some of the RG trajectories satisfy the Yamagishi conditions for fluctuationinduced first order behavior before reaching the IR stable fixed point. Two examples of this are the middle dashed and lower solid curves corresponding to cases with and without QD respectively .

To guarantee the consistency of the above analysis based on the WF $\epsilon$ expansion one needs to check for the Harris criterion [11] which requires the calculation of the critical exponent $\nu$ given by

$$
\nu=\frac{1}{2-\gamma_{2}\left(g^{*}\right)}
$$

where $\gamma_{2}\left(g^{*}\right)$ is the (mass) anomalous dimension. We have calculated $\gamma_{2}(g)$ in the presence of QD:

$$
\gamma_{2}=\frac{N_{F}^{2}+1}{6 N_{F}^{2}} g_{1}+\frac{2 N_{F}^{2}-3}{6 N_{F}^{2}} g_{2}+\frac{1}{4 N_{F}} y^{2}-4 \Delta
$$

and checked for the conditition $\tilde{d} \nu \geq 2$ (the Harris criterion) at the fixed point $\left(g_{1}^{*}, g_{2}^{*}\right)$. Although we find that the Harris criterion is satisfied in the entire region $0 \leq \epsilon, \tilde{\epsilon} \leq 1$, an examination of the RG trajectories (see Fig.1) reveals that as in the pure case, except for a narrow range of parameters in the $\left(N_{C}, N_{F}\right)$ plane, the phase transition is fluctuation induced first order. The occurence of this phenomenon depends on the parameters $N_{C}$ and $N_{F}$ (see Ref. [14 for details on a similar system without impurities) and typically there exists a critical impurity concentration, $\Delta_{\text {crit. }}$, above which the phase transition will be fluctuation induced first order. In the range of parameters where the phase transition is second order, the gauged Yukawa matrix theory with (weak) quenched disorder belongs to 
the universality class of the pure system. Since the specific heat exponent remains neagative, this is expected and further supports the consistency of this work with previous results. The physical quantum spin $j$ antiferromagnet corresponds to the parameter values $N_{F}=2$, $N_{C}=2 j$. With $N_{C}=1(j=1 / 2)$ the non-Abelian field disappears and the resulting theory has no IR fixed point, indicating a first order phase transition. The case $N_{F}=2, N_{C} \geq 2$ (i.e., $j \geq 1$ ) belongs to the asymptotically free regime and hence these antiferromagnets cannot be analyzed by our techniques. In this domain in the $N_{C}, N_{F}$ parameter space we speculate that confinement is associated with a nonperturbative IR fixed point of the gauge coupling. In this case these antiferromagnets are likely to exhibit second order phase transition.

In conclusion, we have shown that long wavelength quantum fluctuations can drive first order phase transitions in the presence of weak quenched disorder when naively none is expected. It is important to note that we have studied the effects of QD on the critical behavior of the pure system by first analyzing the relevance or irrelevance of disorder at the critical fixed point of the pure system and then carrying out a thorough examination of the stability of the pure system against quantum fluctuations. On the other hand, one may also study the crossover from the pure fixed point to the disorder fixed point. This is an important question whose examination, however, is beyond the scope of the present paper. In general, detailed calculations [21] show that when the pure fixed point is unstable (i.e. $\nu d<2$ ) the disorder fixed point is a critical fixed point with new critical exponents [In such instances the stability matrix typically has complex eigenvalues (see, e.g., Dorogovtsev in Ref. [7]), which imply that the RG trajectories spiral into the fixed point basins of attraction, and give oscillatory corrections to scaling]. The results of this paper may help us better understand the nature of phase transitions and the underlying dynamics in low-dimensional systems with weak quenched disorder, such as those occuring in planar high- $T_{C}$ superconductors.

It is a pleasure to thank Michael Ma for invaluable discussions and correspondence which culminated in this work. I would also like to thank T.H. Hansson, E. Langmann and L.C.R. Wijewardhana for thoughtful comments and numerous discussions while this work was being completed.

This work is supported in part by the United States Department of Energy under Grant No. DE-FG02-84ER40153 and by the Swedish Natural Science Research Council.

\section{References}

[1] J. Smit, Nucl. Phys. B175, 307 (1980); T. Banks et al., Phys. Rev. D 15, 1111 (1977).

[2] M. Salmhofer and E. Seiler, Commun. Math. Phys. 139, 395 (1991).

[3] E. Langmann and G. Semenoff, Phys. Lett. 297B, 175 (1992).

[4] M.C. Diamantini, P. Sodano, E. Langmann and G. Semenoff, Nucl. Phys. B406, 595 (1993).

[5] P. Beran, P. Poilblanc and R. B. Laughlin, preprint cond-mat/9505085. 
[6] V. Barzykin and D. Pines, Phys. Rev. B 52, 13585 (1995); S. Chakravarty, B. I. Halperin and D. R. Nelson, Phys. Rev. B 39, 2344 (1989).

[7] A.B. Harris and T.C. Lubensky, Phys. Rev. Lett. 33, 1540 (1974); G. Grinstein and A. Luther, Phys. Rev. B 13, 1329 (1976); A. Aharony in Phase Transitions and Critical Phenomena, edited by C. Domb and M.S. Green (Academic Press, New York, 1976), Vol. VI, p. 357; T.C. Lubensky in Ill-Condensed Matter, edited by R. Balian, R. Maynard and G. Toulouse (North-Holland, Amsterdam, 1979); D.E. Khmelnitskii, Phys. Lett. 67A, 59 (1978); S.N. Dorogovtsev, Phys. Lett. 76A, 169 (1980); T. R. Kirkpatrick and D. Belitz, Phys. Rev. Lett. 76, 2571 (1996).

[8] D. Boyanovsky and J. L. Cardy, Phys. Rev. B 26, 154 (1982).

[9] K.G. Wilson and M.E. Fisher, Phys. Rev. Lett. 28, 240 (1972).

[10] S. Coleman and E. Weinberg, Phys. Rev. D 7, 1888 (1973).

[11] A.B. Harris, J. Phys. C7, 1671 (1974).

[12] N. Read and S. Sachdev, Nucl. Phys. B316, 609 (1989).

[13] See J. Kogut, Rev. Mod. Phys. 55, 775 (1980) for a review.

[14] H. Hamidian, S. Jaimungal, G.W. Semenoff, P. Suranyi and L.C.R. Wijewardhana, Phys. Rev. D 53, 5886 (1996).

[15] H. Hamidian, G.W. Semenoff, P. Suranyi and L.C.R. Wijewardhana, Phys. Rev. Lett. 74, 4976 (1995).

[16] K.G. Wilson, Phys. Rev. D 14, 2911 (1974).

[17] J. Zinn-Justin, Nucl. Phys. B367, 105 (1991); K. Kärkkäinen, R. Lacaxe, P. Lacock and B. Peterson, Nucl. Phys. B415, 781 (1994).

[18] B. Rosenstein, H.L. Yu and A. Kovner, Phys. Lett. B314, 381 (1981).

[19] S.F. Edwards in Polymer Networks, edited by A.J. Chompff and S. Newman (New York, Plenum Press, 1971); P.G. De Gennes, Phys. Lett. A38, 339 (1972); G. Grinstein et al. in [7] above; V.J. Emery, Phys. Rev. B 11, 239 (1975); S.F. Edwards and P.W. Anderson, J. Phys. F5, 965 (1976).

[20] H. Yamagishi, Phys. Rev. D 23, 1880 (1981).

[21] Lubensky in Ref.[7] and A. Weinrib and B.I. Halperin, Phys. Rev. B 27, 413 (1983).

Figure caption: Fig. 1: Projection of the RG trajectories in the six-dimensional coupling constant space onto the $g_{1}-g_{2}$ plane for $N_{C}=2, N_{F}=13$ and $\epsilon=\epsilon_{d}=0.1$. The dashed and solid curves correspond to theories with and without quenched disorder, respectively. The straight dashed line is defined by Eq. (18) and the lowest curve is a runaway trajectory. 


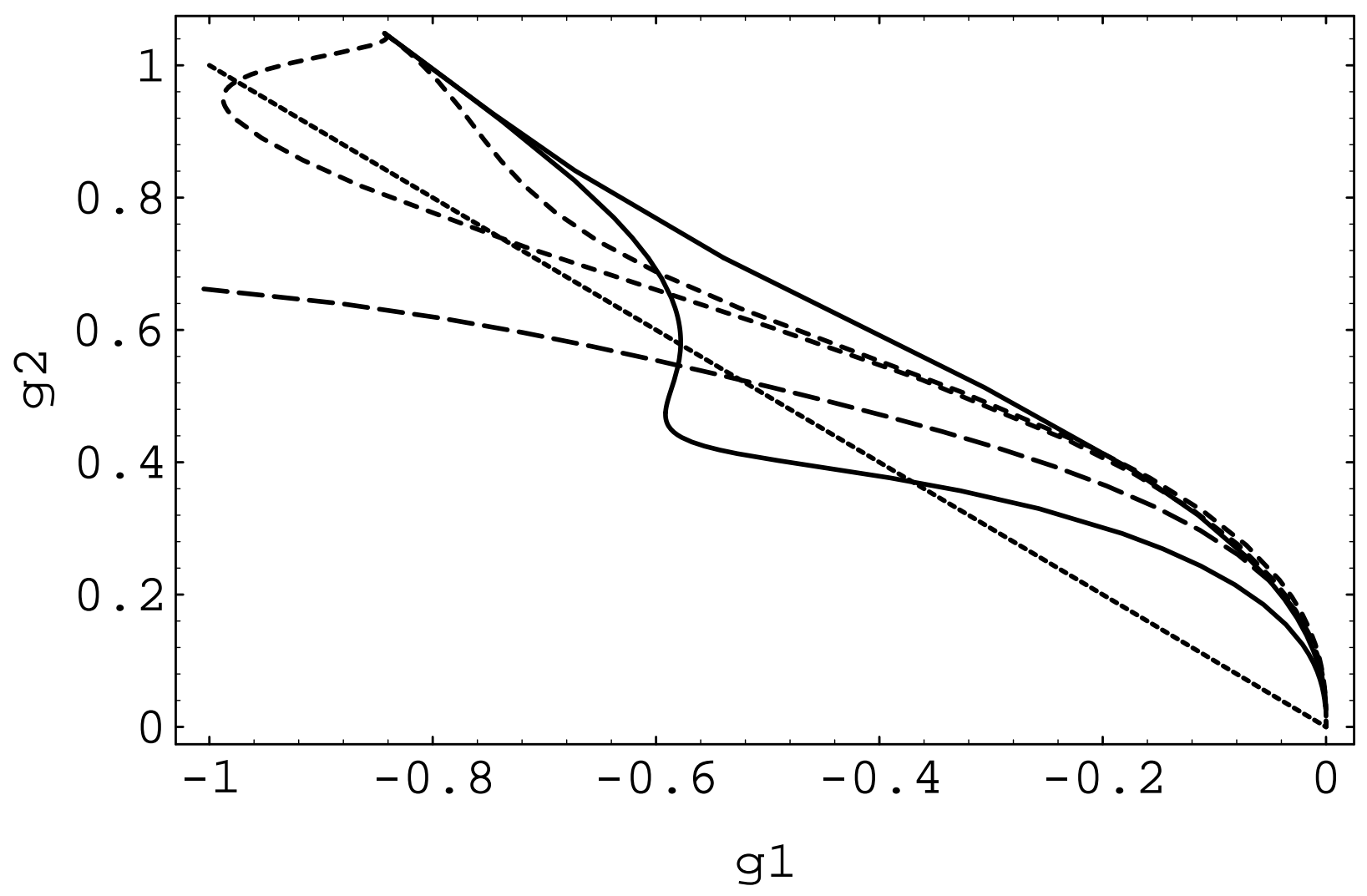

